\title{
Integrability analysis of regular and fractional Blackmore-Samulyak-Rosato fields
}

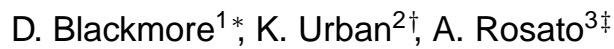 \\ ${ }^{1}$ Department of Mathematical Sciences and Center for Applied Mathematics and Statistics, New Jersey \\ Institute of Technology Newark, NJ 07102-1982 \\ 2 Center for Solar-Terrestrial Research, New Jersey Institute of Technology, Newark, NJ 07102-1982 \\ 3 Department of Mechanical Engineering, New Jersey Institute of Technology, Newark, NJ 07102-1982
}

Received October 11, 2010

\begin{abstract}
Blackmore-Samulyak-Rosato (BSR) fields, originally developed as a means of obtaining reliable continuum approximations for granular flow dynamics in terms of relatively simple integro-differential equations, can be used to model a wide range of physical phenomena. Owing to results obtained for one-dimensional granular flow configurations, it has been conjectured that BSR models of fields with perfectly elastic interactions are completely integrable infinite-dimensional Hamiltonian systems. This conjecture is proved for BSR models in one space dimension, and analogues of BSR fields involving fractional time derivatives are briefly investigated.
\end{abstract}

Key words: BSR model, bi-Hamiltonian, completely integrable, fractional derivative

PACS: $45.70 . \mathrm{Mg}, 47.10 . \mathrm{Df}, 02.30 . \mathrm{k}, 02.60 . \mathrm{Nm}$

\section{Introduction}

Blackmore et al. [5] introduced an (averaged-limiting) integro-partial differential equation continuum approximation for the momentum equation in particulate dynamics that we shall designate as a $B S R$ model; the associated infinite-dimensional vector field shall be called a $B S R$ field, and the method of employing these models shall be referred to as the BSR approach. The primary goal in the development of the BSR models was to find relatively simple and mathematically more tractable models for analyzing and predicting particle dynamics, but they can be readily generalized to obtain a variety of plausible approximate mathematical models for physical fields ranging from micro to subatomic scales.

Another goal of the BSR approach is to provide alternatives for other approximate continuum methods for investigating nearest-neighbor interaction fields comprised of large numbers of components, such as the long-wave approximation, which occupies a special place in the creation and application of soliton theory via a continuum limiting model for Fermi-Pasta-Ulam chains [12] in terms of the Korteweg-de Vries (KdV) equation by Zabusky \& Kruskal [26]. Therefore, it seems likely that analogous connections with completely integrable Hamiltonian systems should also be possible for BSR models, and it is our intention to explore such links in considerable detail in this paper.

The intimate link between approximate continuum models for many-component nonlinear interaction phenomena and infinite-dimensional completely integrable Hamiltonian dynamical systems (with their associated soliton solutions) established for the long-wave method and indicated for the BSR approach has manifested itself in numerous applications, several of which have arisen in the investigation of granular flows. For example, Nesterenko [17] showed that certain long-wave approximations of perfectly elastic (Hertzian) one-dimensional particle dynamics can be transformed

\footnotetext{
*E-mail: deblac@m.njit.edu

†E-mail: kdu2@njit.edu

${ }_{\ddagger}^{\ddagger}$ E-mail: anthony.rosato@njit.edu
} 
into KdV equations. On the other hand, Blackmore \& Dave [4] proved that perfectly elastic onedimensional particle dynamics modeled by a BSR field can be approximated by Burgers' equation, which is also completely integrable. In particular, the results in [4] led to the conjecture that BSR field approximations to perfectly elastic (non-dissipative) granular flows are completely integrable. And as BSR approximations can be generalized to apply to a much broader class of physical phenomena, the conjecture should be extended to include BSR continuum models of more general non-dissipative fields. A proof of this conjecture is the main result of this paper.

When it comes to field equations of practically every kind, a recent - and rather productive - trend has been to reformulate the associated classical differential equation models in terms of fractional derivatives, and compare the solutions of the fractional and classical versions. These fractional versions of classical field equations have yielded some very interesting and useful results, which include certain types of intrinsic gauge invariance and systemic dissipation effects in fractional forms of non-dissipative classical fields. With this in mind, we shall briefly consider analogues of BSR fields having fractional Caputo time derivatives. Our main observation is that fractional near integral derivatives with respect to time tend to introduce dissipation in classically inelastic systems - an observation seen in many fractionalized classical field equations.

Our treatment begins in section 2 with a terse introduction to generalized BSR field equations. In section 3, we follow this with our main result that the BSR fields satisfying certain mild assumptions are completely integrable infinite-dimensional Hamiltonian dynamical systems, which is proved by demonstrating that the fields in question have a rather simple bi-Hamiltonian structure. Then in section 4, we consider fractional derivative reformulations of our BSR fields and show by example that the resulting dynamical systems include dissipative effects that render them nonintegrable. We conclude our investigation in section 5 with some remarks on the results of our analysis here and a brief indication of related future research.

\section{BSR field equations}

Our original derivation of the BSR model [5] was formulated in the context of granular flows in one-, two- or three-dimensional Euclidean space, denoted as usual by $\mathbb{R}, \mathbb{R}^{2}$ or $\mathbb{R}^{3}$, respectively, where the particle-particle and particle-boundary forces were postulated to be of a form general enough to subsume the usual Hertz-Mindlin and Walton-Braun models (cf. [6, 17, 23, 23 25] ). It took the form of the following integro-partial differential equation (IPDE):

$$
u_{t}+\sum_{j=1}^{m} u_{j} u_{x_{j}}=E(x, t)+\int_{\mathbb{R}^{m}} \Theta(x, y, t, u(x+y, t)-u(x, t) ; \mu) \mathrm{d} y,
$$

where $x, y \in \mathbb{R}^{m}, m=1,2,3, t$ represents time, $u=\left(u_{1}, u_{2}, u_{3}\right)=\dot{x}$ is the velocity of a particle moving through $\mathbb{R}^{m}$, the variable subscripts denote partial derivatives, $(x, t)$ is a point in the $(m+1)$ dimensional spacetime $\mathbb{R}^{m} \times \mathbb{R}, E$ represents the external force on the flow configuration that is often a constant, the integral is the standard Lebesgue integral, the kernel $\Theta$ is a smooth function that vanishes for $|y|$ sufficiently large uniformly in the other variables in accordance with the assumed nearest-neighbor or nearest-boundary interaction assumptions for the particle dynamics, and $\mu$ represents a vector of parameters defined by the physical properties of the constituents of the granular flow.

If one examines the limiting argument used to obtain (1) in the granular flow context, it is easy to see that it generalizes quite naturally to obtain approximate continuum models for any physical dynamical system on any smooth finite-dimensional manifold $M$ comprised of a very large number of particles - possibly acting upon one another and their environment in both near-neighbor and at a distance action modes. Then, the resulting general BSR field model takes the form

$$
u_{t}+\nabla_{u} u=E(x, t)+\int_{M} \Theta\left(x, y, t, u_{\backslash x}(y, t)-u(x, t) ; \mu\right) \mathrm{d} y,
$$

where $\nabla_{u}$ denotes the usual covariant derivative (for fixed $t$ ) on the Riemannian manifold $M, \mathrm{~d} y$ is the standard Lebesgue measure based differential associated with integration on $M$ and $u_{\backslash x}$ 
is the parallel transport of the vector $u(y, t)$ along a geodesic from $y$ to $x$ on $M$. Everything in (2) is assumed to be smooth (i.e. $C^{\infty}$ ) with the integrand and all of its derivatives going to zero exponentially fast as the (geodesic) distance from $y$ to any fixed $x$, denoted as $\varrho(x, y)$, increases without bound. To simplify matters, we shall assume that the field is defined on the flat manifold $\mathbb{R}^{m}$, so that the field equations that we are going to consider in the sequel have the form

$$
u_{t}+\nabla_{u} u=E(x, t)+\int_{\mathbb{R}^{m}} \Theta(x, y, t, u(y, t)-u(x, t) ; \mu) \mathrm{d} y
$$

where

$$
\nabla_{u} u=\sum_{j=1}^{m} u_{j} u_{x_{j}}
$$

for any natural number $m$ in $\mathbb{N}$ and $d y$ denotes the standard $m$-dimensional Lebesgue measure.

It should be noted that (3) can also be approximated as a partial differential equation (PDE) obtained by expanding $\Theta$ in Taylor series (about $(x, t)$ ), integrating and then truncating the series after a suitable finite number of terms (cf. [4, 5]), but we shall not consider these approximations in any detail in the sequel.

\section{Integrability analysis of BSR system}

Integrability analysis has played a vital role in the development and investigation of approximate continuum models for systems of very large numbers of interacting components. In fact, one may argue persuasively that soliton theory and integrability of infinite-dimensional Hamiltonian dynamical systems is a direct result of such approximations. In particular, the work of Zabusky \& Kruskal [26] in showing that the long-wave limit of an FPU chain [12] of interacting nonlinear oscillators is equivalent to the completely integrable $\mathrm{KdV}$ equation served as the catalyst for the spectacular advances in the theory and applications of infinite-dimensional Hamiltonian systems during the last half-century, recorded and applied in such works as [1, 7 11, 14, 17 21].

From the perspective of the origins of (3), the demonstration of soliton-like behavior for longwave limits of one-dimensional particle chains interacting in a perfectly elastic manner [17] and the integrability of PDE approximations of the BSR model [4] for the same kinds of chains strongly suggest that the corresponding dynamical systems are completely integrable Hamiltonian systems. We shall prove this here for (3) in one-space dimension $(m=1)$ when the interactions are perfectly elastic (non-dissipative). Analogous integrability results can be obtained for any finite space dimension, but the modifications necessary to deal with the higher dimensional equations are quite extensive, so we shall defer a more general treatment to a forthcoming study.

Our investigations [4 6] have revealed that perfectly elastic particle-particle and particle-boundary interactions are synonymous with the kernel in (3) being independent of $u(y, t)-u(x, t)$, so that the BSR equation assumes the simpler form

$$
u_{t}+\nabla_{u} u=F(x, t ; \mu):=E(x, t)+\int_{\mathbb{R}^{m}} \Theta(x, y, t ; \mu) \mathrm{d} y,
$$

where $F$ is a smooth function. Hence, for our investigation here, we take our (space dimension one) perfectly elastic BSR continuum model to be the above equation with $m=1$.

To see the plausibility of the complete integrability of (4), let us look for a traveling-wave solution of the following special case of the governing equation:

$$
\frac{\partial u}{\partial t}+u \frac{\partial u}{\partial x}=\varphi(x-V t)
$$

where $\varphi: \mathbb{R} \rightarrow[0, \infty)$ is a smooth (nonnegative) absolutely (Lebesgue) integrable function on $\mathbb{R}$. We seek a solution of the form

$$
u=f(\xi)=f(x-V t),
$$

which we substitute in (4) to obtain

$$
-V f^{\prime}+f f^{\prime}=\varphi(\xi)
$$


Then one integration yields the solutions

$$
u=V \pm \sqrt{V^{2}+2 \Phi(x-V t)}
$$

where $\Phi$ is simply the integral of $\varphi$. We also see that the form of the wave is essentially completely determined by the force field $\varphi(x-V t)$.

\subsection{Hamiltonian form of the nondissipative field for $m=1$}

As mentioned above, we shall investigate the Hamiltonian structure and integrability of the perfectly elastic BSR field in one space dimension, which assumes the form

$$
u_{t}+u u_{x}=F(x, t ; \mu)
$$

where $(x, t) \in \mathbb{R} \times[0, \infty), \mu$ is a parameter (vector) in $\mathbb{R}^{k}$, and $F$ is a smooth function on its domain of variables and parameters. First, we attend to the Hamiltonian structure and the identification of the associated Hamiltonian function. The simplest Hamiltonian representation for (6) is

$$
u_{t}=\theta \circ \nabla H
$$

where

$$
H:=\int_{\mathbb{R}}\left(-\frac{1}{6} u^{3}+u \partial_{x}^{-1} F\right) \mathrm{d} x,
$$

$\nabla$ is the usual (variational) gradient, and $\theta=\partial_{x}$, where $\partial_{x}$ is the standard partial derivative operator with respect to $x$. For our proof of integrability we are going to find a different compatible Hamiltonian representation that guarantees that there is an infinite hierarchy of independent invariants for (7) in virtue of Magri's theorem and its various improvements (cf. [7, 8, 16]), which we take as complete integrability. In particular, we have the following result with a very straightforward proof.

Theorem 1 Suppose that all of the functions in (6) are in the usual Schwarz space of functions (depending on the number of their variables and parameters) and that $F$ does not depend explicitly on time, i.e. $F(x, t ; \mu)=F(x ; \mu)$. Then the infinite-dimensional Hamiltonian dynamical system (7) representing (6) is completely integrable in the sense that it possesses an infinite hierarchy of constants of motion

$$
H_{0}:=H, H_{1}, H_{2}, \ldots
$$

that are in involution with respect to the usual Poisson bracket induced by $\theta$, namely

$$
\{f, g\}(u)=\{f, g\}_{\theta}(u):=\langle\nabla g, \theta \circ \nabla f\rangle(u)=L_{\theta \circ \nabla f} g(u)
$$

where $\langle\cdot, \cdot\rangle$ is the standard scalar (inner) product and $L$ is the Lie derivative (cf. [8]).

Proof. It follows from refinements of Magri's theorem (see [7, 8]) that it suffices to find another Hamiltonian representation of the system (7) in the form

$$
u_{t}=\hat{\theta} \circ \nabla \hat{H}
$$

where the Poisson (implectic) operator pair $(\theta, \hat{\theta})$ is compatible. For completely integrable systems there is naturally a denumerable choice of compatible bi-Hamiltonian representations, and it suffices to find just one of the form (9) having the necessary properties.

For (9) we choose

$$
\hat{\theta}:=-u \partial_{x} u+F \text { and } \hat{H}:=u .
$$

Then it is a simple matter to verify the compatibility of the representations (7) and (9), from which the complete integrability follows, thereby concluding the proof. 
One can use the details of the proof to construct an infinite hierarchy of integrals of (6) using the recursion techniques developed to deal with multi-Hamiltonian systems, but there is in fact a much simpler way to find integrals: Start by simply writing (6) in the conservation form

$$
(u)_{t}+\left(-\frac{1}{2} u^{2}+\partial_{x}^{-1} F\right)_{x}=0
$$

which, under the Schwarz space assumption, leads immediately to the conclusion that

$$
\int_{\mathbb{R}} u \mathrm{~d} x
$$

is a constant of motion, which is - not surprisingly - tantamount to conservation of mass. Then multiply (6) by $u$ to obtain the following equation written in conservation form:

$$
\left(\frac{u^{2}}{2}\right)_{t}+\left(-\frac{1}{3} u^{3}+\partial_{x}^{-1}(u F)\right)_{x}=0
$$

from which we obtain the constant of motion corresponding to conservation of momentum, namely

$$
\int_{\mathbb{R}} \frac{u^{2}}{2} \mathrm{~d} x
$$

Continuing in this fashion, we find that all of the functions

$$
\int_{\mathbb{R}} \frac{u^{n}}{n+1} \mathrm{~d} x, \quad n=1,2, \ldots
$$

are constants of motion.

\section{Fractional BSR models}

We shall now touch briefly on a particularly simple fractional derivative analogue of the BSR field model involving only Reisz-Caputo fractional derivatives in time. As mentioned in the introduction, there is a very active current trend of revisiting fractional derivative forms of classical dynamical systems representations of phenomena arising in physics and other fields in order to compare the behaviors of the classical and fractional models (examples of which can be found in $[2,[3,[13])$.

The fractional derivative analogue of (6), when $F$ does not depend explicitly on time, that we consider is

$$
\mathfrak{d}_{t}^{\alpha} u+u u_{x}=F(x ; \mu)
$$

where $\mathfrak{d}_{t}^{\alpha}$ is the (partial) Riesz-Caputo derivative of order $\alpha$ (taken to be any real number close to 1) defined as

$$
\mathfrak{d}_{t}^{\alpha} f(x, t):=\frac{1}{\Gamma(n-\alpha)} \int_{0}^{\infty}|t-\tau|^{n-\alpha-1}\left(\frac{\partial}{\partial \tau}\right)^{n} f(\tau) \mathrm{d} \tau
$$

and $\Gamma$ is the usual Gamma function. To show that such an equation can actually be extracted from first principles of a sort, we can consider a fractional variational approach. Following the approach for obtaining the Euler-Lagrange equations and then the corresponding Hamiltonian formulation for a Lagrangian involving fractional derivatives (as in [2, 3] ) - in this case only a fractional RieszCaputo derivative of $u$ with respect to time - one can obtain (12) in a more natural way consistent with the fundamental principles of mechanics.

Our preliminary analysis of (12) shows that non-integer values of the time derivative inherently produce dissipative effects, just as can readily be shown via an exact solution in the case of the fractional linear oscillator

$$
\mathfrak{D}_{t}^{\alpha} \varphi+\varphi=0
$$

for non-integral values of $\alpha$ near two. Here $\mathfrak{D}_{t}^{\alpha}$ represents the ordinary Riesz-Caputo derivative of order $\alpha$. The inference (based so far only on limited evidence) is that truly integrable behavior may not be possible for (12) when $\alpha$ is not an integer. However, it may be possible to obtain solutions in traveling wave type form for some cases. 


\section{Epilogue}

We have shown that the BSR equation in one space dimension is an infinite-dimensional completely integrable Hamiltonian dynamical system when it does not depend explicitly on time. This also appears to be the case for higher space dimensions, and we intend to address this in a forthcoming paper, where we shall present a detailed investigation of solitons and other wave-like phenomena. These results will be compared with the experimental findings on various granular flow regimes such as the types in Rosato et al. 22, 23] in accordance with our continuing project on the development of dynamical systems based methods for predicting particulate dynamics (cf. [6]).

Another area of possible research along the lines presented here concerns the case where the BSR model involves additional terms that represent small perturbations from a completely integrable form. If this perturbed system is still Hamiltonian, we shall want to see which of the existing infinite-dimensional KAM theorems are applicable (see e.g. [14]) and perhaps prove some new results of this kind that can be used to gain further insight into the nature of more general BSR dynamics. When the perturbation is non-Hamiltonian, it may still be possible to extend some of the methods employed in Prykarpatsky et al. [20] and other related studies to characterize certain invariant aspects of BSR dynamics. We plan to look into each of these questions in the near future.

The preliminary findings on fractional analogues of BSR fields suggest that a more thorough study of such systems is in order. We shall in the near future investigate (12) in much greater detail, and also delve into what are perhaps more realistic fractional analogues in which the actual interaction forces in the particle fields are fundamentally fractional in nature as for example in [15].

\section{Acknowledgement}

The authors wish to thank the National Science Foundation for partial support of the research in this paper under NSF Grant CMMI-1029809. Thanks are also due to Anatoliy Prykarpatsky for his constant support and indispensable advice on all matters relating to integrability analysis - and especially for the work here.

\section{References}

1. Ablowitz M., Segur H., Solitons and the Inverse Scattering Transform. SIAM, Philadelphia, 1981.

2. Agarwal P., Comp. Math. Appl., 2010, 59, 1852-1864.

3. Baleanu D., Rep. Math. Phys., 2008, 61, 199-206.

4. Blackmore D., Dave R., Chaos in One-Dimensional Granular Flows with Oscillating Boundaries. Powders \& Grains 97, R. Behringer and J. Jenkins (eds.). Balkema, Rotterdam, 1997, p. 409-412.

5. Blackmore D., Samulyak R., Rosato A., J. Nonlin. Math. Phys., 1999, 6, 198-221.

6. Blackmore D., Rosato A., Tricoche X., Urban K., Ratnaswamy V., Tapping dynamics for a column of particles and beyond, J. Mech. Materials and Structures, (to appear).

7. Blackmore D., Prykarpatsky A., Samoylenko V., Nonlinear Dynamics of Mathematical Physics: Spectral and Symplectic Integrability Analysis. World Scientific, New Jersey (in press).

8. Błasek M., Multi-Hamiltonian Theory of Dynamical Systems. Springer- Verlag, Berlin, 1998.

9. Bogolubov (Jr.) N.N., Blackmore D., Samoylenko V., Prykarpatsky, A., Opuscula Mathematica, 2007, 27, 187-195.

10. Dickey L., Soliton Equations and Hamiltonian Systems. World Scientific, Singapore, 1991.

11. Fadeev L., Takhtajan L., Hamiltonian Methods in the Theory of Solitons. Springer-Verlag, Berlin, 1987.

12. Fermi E., Pasta J., Ulam S., Collected Works of Enrico Fermi, Vol. 2. Chicago University Press, Chicago, 1965.

13. Gafiychuk V., Datsko B., Meleshko V., Blackmore D., Chaos, Solitons and Fractals, 2009, 41, 10951104.

14. Kuskin S., Nearly Integrable Infinite-dimensional Hamiltonian Systems, Lecture Notes in Mathematics, Vol. 1556. Springer-Verlag, New York, 1993.

15. Leszczynski J., Phys. Rev. E, 2004, 70, 051315.

16. Magri F., J. Math. Phys., 1978, 19, 1156-1162. 
17. Nesterenko V., J. Appl. Mech. Tech. Phys., 1984, 24, 733-743.

18. Prykarpatsky A., Mykytiuk I., Algebraic Integrability of Nonlinear Dynamical Systems on Manifolds: Classical and Quantum Aspects. Kluwer, Dordrecht, 1998.

19. Prykarpatsky A., Blackmore D., Bogolubov (Jr.) N.N., J. Open Systems \& Information Dynamics, 1999, 6, 335-373.

20. Prykarpatsky Y., Samoilenko A.M., Blackmore D., Rep. Math. Phys., 1999, 44, 171-182.

21. Prykarpatsky Y., Samoilenko A., Prykarpatsky A., Bogolubov (Jr.) N.N., Blackmore D., The Differential-Geometric Aspects of Integrable Dynamical Systems. Preprint \#IC/2007/030 of the Abdus Salam International Centre for Theoretical Physics, Trieste, Italy.

22. Rosato A., Ratnaswamy V., Horntrop D., Dybenko O., Kondic L., Temporal dynamics in density relaxation, Proc. Joint IUTAM-ISIMM Sympos. on Mathematical Modeling and Physical Instances of Granular Flows, Reggio-Cabria, Italy, 14-18 Sept. 2009, AIP Conference Proceedings 1227, p. 89-99.

23. Rosato A., Dybenko O., Ratnaswamy V., Horntrop D., Kondic L., Phys. Rev. E, 2010, 81, 061301.

24. Savage S., Adv. Appl. Mech., 1984, 24, 289-366.

25. Walton O., Numerical simulation of inelastic, frictional particle-particle interactions, in Particulate Two-phase Flow, M.C. Rocco (ed.). Butterworth-Heinemann, Boston, 1992, p. 884-911.

26. Zabusky N., Kruskal M., Phys. Rev. Lett., 1965, 15, 240-243.

\title{
Аналіз інтегровності регулярних і функціональних BSR полів
}

\author{
Д. Блекмор 11, К. Урбан², А. Росато ${ }^{3}$ \\ 1 Факультет математичних наук і Центр прикладної математики і статистики, Інститут технології Нью \\ Джерсі, Ньюарк, США \\ 2 Центр сонячно-земних досліджень, Інститут технології Нью Джерсі, Ньюарк, США \\ 3 Інженерно-механічний факультет, Інститут технології Нью Джерсі, Ньюарк, США
}

BSR поля, початково розвинуті як засіб отримання надійних континуумних апроксимацій для динаміки гранулярних потоків на мові відносно простих інтегро-диференціальних рівнянь, можуть бути використані для моделювання широкого спектру фізичних явищ. Завдяки результатам, отриманим для конфігурацій одновимірного гранулярного потоку, було зроблено припущення що BSR моделі полів з ідеально пружними взаємодіями є повністю інтегровними нескінченновимірними гамільтоновими системами. Це припущення $€$ доведеним для BSR моделей в одновимірному просторі, і аналоги BSR полів, що включають часткові похідні за часом є стисло дослідженні.

Ключові слова: BSR модель, бі-гамільтоніан, повністю інтегровний, часткова похідна 
